\title{
Fermionic behavior of ideal anyons
}

\author{
Douglas Lundholm $^{1}$ (D) Robert Seiringer $^{2}$
}

Received: 23 February 2018 / Accepted: 24 April 2018 / Published online: 11 May 2018

(C) The Author(s) 2018

\begin{abstract}
We prove upper and lower bounds on the ground-state energy of the ideal two-dimensional anyon gas. Our bounds are extensive in the particle number, as for fermions, and linear in the statistics parameter $\alpha$. The lower bounds extend to LiebThirring inequalities for all anyons except bosons.
\end{abstract}

Keywords Intermediate quantum statistics · Magnetic interaction · Ideal anyon gas · Lieb-Thirring inequality

Mathematics Subject Classification 81 V70 $\cdot 81 \mathrm{Q} 10 \cdot 35 \mathrm{P} 15 \cdot 46 \mathrm{~N} 50$

\section{Introduction}

The behavior of quantum mechanical systems of particles depends sensitively on the geometry of the space in which the particles may move. In particular, dimensionality plays a significant role, and it is a geometric fact that only two fundamental types of identical particles naturally occur in three-dimensional space-bosons and fermions, from whose basic statistical properties many collective quantum phenomena follow. More exotic possibilities of quantum statistics may be realized by confining the particles' motion and thereby effectively lowering the dimensionality. In two spatial

\footnotetext{
$\triangle$ Douglas Lundholm dogge@math.kth.se

Robert Seiringer robert.seiringer@ist.ac.at

1 Department of Mathematics, KTH Royal Institute of Technology, 10044 Stockholm, Sweden

2 IST Austria, Am Campus 1, 3400 Klosterneuburg, Austria
} 
dimensions, which we will be concerned with here, the richer topology allows for a family of hypothetical quantum particles known as anyons.

Recall that the state of a quantum system of $N$ particles is described in terms of a Schrödinger wave function, $\Psi:\left(\mathbb{R}^{2}\right)^{N} \rightarrow \mathbb{C}$, whose amplitude $|\Psi(\mathrm{x})|^{2}$ represents the probability density of finding the particles at positions $\mathrm{x}=\left(\mathbf{x}_{1}, \ldots, \mathbf{x}_{N}\right), \mathbf{x}_{j} \in \mathbb{R}^{2}$. If the particles are indistinguishable, one must impose that the density is symmetric under particle exchange, i.e.,

$$
\left|\Psi\left(\mathbf{x}_{1}, \ldots, \mathbf{x}_{j}, \ldots, \mathbf{x}_{k}, \ldots, \mathbf{x}_{N}\right)\right|^{2}=\left|\Psi\left(\mathbf{x}_{1}, \ldots, \mathbf{x}_{k}, \ldots, \mathbf{x}_{j}, \ldots, \mathbf{x}_{N}\right)\right|^{2}, \quad j \neq k
$$

This leaves the possibility for an exchange phase:

$$
\Psi\left(\mathbf{x}_{1}, \ldots, \mathbf{x}_{j}, \ldots, \mathbf{x}_{k}, \ldots, \mathbf{x}_{N}\right)=e^{i \alpha \pi} \Psi\left(\mathbf{x}_{1}, \ldots, \mathbf{x}_{k}, \ldots, \mathbf{x}_{j}, \ldots, \mathbf{x}_{N}\right), \quad j \neq k
$$

In the case of bosons $(\alpha=0)$ or fermions $(\alpha=1)$, one has $e^{i \alpha \pi}= \pm 1$, so that a double exchange is trivial. However, by clarifying in topological terms what exactly should be meant by the exchange (1.1) (say a simple counterclockwise continuous exchange of two particles), it is possible to allow for any phase $e^{i \alpha \pi} \in U(1)$ or statistics parameter $\alpha \in \mathbb{R}$, thereby defining a system of anyons. ${ }^{1}$ Such possibilities have been known since the 1970s and have been studied extensively in the physics literature during the following decades, with notable proposals for concrete realizations and applications, such as for quasi-particles in the fractional quantum Hall effect, rotating cold quantum gases, as well as for future prospects of quantum information storage and computation. We refer to [3,7,9-11,13,23,29-33] for reviews.

Mathematically, anyons can be realized by viewing $\Psi$ as a multi-valued function or a section of a complex line bundle over a nontrivial configuration manifold, an approach known in the literature as the anyon gauge picture [4]. Alternatively, one can start with the usual quantum-mechanics setup, taking the familiar bosons or fermions as a reference system, and adding to these magnetic interactions of Aharonov-Bohm type $[22,24,26]$. Here we shall follow this latter approach, known as the magnetic gauge picture.

Many basic questions concerning the behavior of many-particle systems of anyons have remained open since their discovery. This is true even for ideal anyons, i.e., particles without any interactions in addition to the ones forced by statistics. While noninteracting bosons and fermions admit a description solely in terms of the spectrum and eigenstates of the corresponding one-body problem, allowing for the properties of the ideal quantum Bose and Fermi gases to be worked out easily, anyons with $0<\alpha<1$ do not admit such a simplification and must be treated within the full many-body context. Even their ground-state properties are thus difficult to determine. In contrast, recall that ideal bosons at zero temperature display complete Bose-Einstein condensation into a single one-body state of lowest energy, while fermions are distributed over the $N$ lowest one-body states to satisfy the Pauli exclusion principle, leading in particular to the extensivity of the fermionic ground-state energy.

\footnotetext{
1 More precisely, these are abelian anyons. Non-abelian anyons may be defined by replacing complex phase factors by unitary matrices $[9,30]$.
} 
We show in this work that the ground-state energy of the ideal anyon gas has a similar extensivity as the one for fermions, for all values of $\alpha$ except for zero (i.e., bosons). In fact, we shall derive upper and lower bounds that interpolate linearly in $\alpha$ between bosons at $\alpha=0$ and fermions at $\alpha=1$. This improves on previous results which only applied to particular rational values of $\alpha$. Via well-known methods, our new bounds imply that also the celebrated Lieb-Thirring inequality holds for all anyons except for bosons.

\section{Model and main results}

In the magnetic gauge formulation, the kinetic energy operator for $N$ ideal (i.e., pointlike) anyons in $\mathbb{R}^{2}$ with statistics parameter $\alpha \in \mathbb{R}$ is given by ${ }^{2}$

$$
\hat{T}_{\alpha}:=\sum_{j=1}^{N} D_{j}^{2}
$$

with the magnetically coupled momenta

$$
D_{j}:=-i \nabla_{\mathbf{x}_{j}}+\alpha \mathbf{A}_{j}, \quad \mathbf{A}_{j}:=\sum_{\substack{k=1 \\ k \neq j}}^{N}\left(\mathbf{x}_{j}-\mathbf{x}_{k}\right)^{-\perp},
$$

where

$$
\mathbf{x}^{-\perp}:=\frac{\mathbf{x}^{\perp}}{|\mathbf{x}|^{2}}=\frac{(-y, x)}{x^{2}+y^{2}} \quad \text { for } \mathbf{x}=(x, y) \in \mathbb{R}^{2}
$$

is the magnetic potential of an Aharonov-Bohm flux of magnitude $2 \pi$ at the origin, satisfying curl $\mathbf{x}^{-\perp}=2 \pi \delta_{0}(\mathbf{x})$. Since we demand that $\alpha=0$ represents bosons in accordance with (1.1), we take the $N$-particle Hilbert space to be $\mathcal{H}=L_{\text {sym }}^{2}\left(\mathbb{R}^{2 N}\right)$, the permutation-symmetric square-integrable functions. The operators $\mathrm{D}_{\alpha}=\left(D_{j}\right)_{j=1}^{N}$ and $\hat{T}_{\alpha}$ then act as unbounded operators on $\mathcal{H}$ and, because of the singular nature of the vector potentials $\mathbf{A}_{j} \notin L_{\text {loc }}^{2}$, some care is needed to properly define their domains. One can in fact show [26, Theorem 5] that on $\mathbb{R}^{2 N}$ the minimal and maximal realizations of $\mathrm{D}_{\alpha}$ coincide and hence induce a natural form domain $\mathscr{D}_{\alpha}^{N}=\operatorname{dom}\left(\mathrm{D}_{\alpha}\right) \subset \mathcal{H}$ for the kinetic energy $\hat{T}_{\alpha}$. This choice is then taken to model ideal anyons. Indeed $\alpha=0$ yields free bosons, while $\alpha=1$ corresponds to fermions, with their domains being the Sobolev spaces $\mathscr{D}_{0}^{N}=H_{\text {sym }}^{1}, \operatorname{dom}\left(\hat{T}_{0}\right)=H_{\text {sym }}^{2}$ and $\mathscr{D}_{1}^{N}=U^{-1} H_{\text {asym }}^{1}, \operatorname{dom}\left(\hat{T}_{1}\right)=$ $U^{-1} H_{\text {asym }}^{2}$, respectively. Here, the unitary map $U: L_{\text {sym/asym }}^{2} \rightarrow L_{\text {asym/sym }}^{2}$,

$$
(U \Psi)\left(\mathbf{x}_{1}, \ldots, \mathbf{x}_{N}\right):=\prod_{1 \leq j<k \leq N} \frac{z_{j}-z_{k}}{\left|z_{j}-z_{k}\right|} \Psi\left(\mathbf{x}_{1}, \ldots, \mathbf{x}_{N}\right), \quad z_{j}:=x_{j}+i y_{j}
$$

\footnotetext{
${ }^{2}$ We choose units such that $\hbar=1=2 m$, with $m$ the particle mass.
} 
transforms bosons with attached unit magnetic flux into free fermions, and vice versa.

In general, the gauge equivalence

$$
\mathrm{D}_{\alpha+2 n}=U^{-2 n} \mathrm{D}_{\alpha} U^{2 n}, \quad \mathscr{D}_{\alpha+2 n}^{N}=U^{-2 n} \mathscr{D}_{\alpha}^{N}, \quad n \in \mathbb{Z},
$$

with $U^{2 n}: \mathcal{H} \rightarrow \mathcal{H}$, implies that the entire spectrum of $\hat{T}_{\alpha}$ is $2 \mathbb{Z}$-periodic in $\alpha$. It is also symmetric under the reflection $\alpha \mapsto-\alpha$, by complex conjugation $\Psi \mapsto \bar{\Psi}$. Note that these properties are all in line with the periodicity of the exchange phase (1.1). In particular, it suffices to consider the case $0 \leq \alpha \leq 1$ only, which we will do from now on.

When restricting to finite domains $\Omega \subset \mathbb{R}^{2}$ the operator $\hat{T}_{\alpha}$ and its spectrum depends on the choice of boundary conditions. We may naturally define a Neumann realization via the nonnegative quadratic form

$$
\left\langle\Psi, \hat{T}_{\alpha}^{\Omega, \mathcal{N}} \Psi\right\rangle=\sum_{j=1}^{N} \int_{\Omega^{N}}\left|D_{j} \Psi\right|^{2}, \quad \Psi \in \mathscr{D}_{\alpha}^{N},
$$

and a Dirichlet realization $\hat{T}_{\alpha}^{\Omega, \mathcal{D}}$ by considering the same form for $\Psi \in \mathscr{D}_{\alpha}^{N}$ with compact support in $\Omega$. In particular, let us define the Neumann ground-state energy for $N$ anyons on a domain $\Omega \subset \mathbb{R}^{2}$ as

$E_{N}^{\mathcal{N}}(\alpha ; \Omega):=\inf \operatorname{spec} \hat{T}_{\alpha}^{\Omega, \mathcal{N}}=\inf \left\{\sum_{j=1}^{N} \int_{\Omega^{N}}\left|D_{j} \Psi\right|^{2}: \Psi \in \mathscr{D}_{\alpha}^{N}, \int_{\Omega^{N}}|\Psi|^{2}=1\right\}$

and likewise for the Dirichlet ground-state energy $E_{N}^{\mathcal{D}}(\alpha ; \Omega)=\inf \operatorname{spec} \hat{T}_{\alpha}^{\Omega, \mathcal{D}}$.

For the special case of $\Omega$ equal to the unit square $Q_{0}=[0,1]^{2}$, we will drop $\Omega$ in the notation for simplicity, and simply write $E_{N}^{\mathcal{N}}(\alpha)$ and $E_{N}^{\mathcal{D}}(\alpha)$, respectively. Note that for a general square $Q \subset \mathbb{R}^{2}$, we have

$$
E_{N}^{\mathcal{N} / \mathcal{D}}(\alpha ; Q)=|Q|^{-1} E_{N}^{\mathcal{N} / \mathcal{D}}(\alpha),
$$

due to the homogeneous scaling property of $\mathrm{D}_{\alpha}$. In particular, in the thermodynamic limit $N \rightarrow \infty,|Q| \rightarrow \infty$ with the density $\rho=N /|Q|$ of the gas kept fixed, the energy per particle is equal to $\rho$ times an $\alpha$-dependent constant, given by $\lim _{N \rightarrow \infty} N^{-2} E_{N}^{\mathcal{N} / \mathcal{D}}(\alpha)$.

The case $\alpha=1$ corresponds to ideal fermions, where the ground state energy is obtained by simply adding up the $N$ lowest eigenvalues of the one-body operator, i.e., the Laplacian $-\Delta_{Q_{0}}^{\mathcal{N} / \mathcal{D}}$. From the Weyl asymptotics, one obtains

$$
E_{N}^{\mathcal{N} / \mathcal{D}}(1)=2 \pi N^{2}+o\left(N^{2}\right) \text { as } N \rightarrow \infty .
$$


On the other hand, for ideal bosons, i.e., $\alpha=0$,

$$
E_{N}^{\mathcal{N}}(0)=0 \quad \text { and } \quad E_{N}^{\mathcal{D}}(0)=2 \pi^{2} N
$$

which equals $N$ times the infimum of the spectrum of the Laplacian $-\Delta_{Q_{0}}^{\mathcal{N} / \mathcal{D}}$. In the case $0<\alpha<1$ of proper anyons, there is no simplification to a one-body problem, however; the system must be treated as a fully interacting many-body system.

Our main result is to show that for anyons with $0<\alpha<1$ and confined to the unit square, $E_{N}^{\mathcal{N} / \mathcal{D}}(\alpha) \sim N^{2}$, as in the fermionic case, with a prefactor that is of order $\alpha$ both in the upper and lower bounds. In this sense, the ideal anyon gas behaves fermionic, for any $\alpha>0$. Since $E_{N}^{\mathcal{D}}(\alpha) \geq E_{N}^{\mathcal{N}}(\alpha)$, it is natural to derive an upper bound on $E_{N}^{\mathcal{D}}(\alpha)$ and a lower bound on $E_{N}^{\mathcal{N}}(\alpha)$.

Our main result is as follows:

Theorem 2.1 (Bounds for the ideal anyon gas) There exist constants $0<C_{1} \leq C_{2}<$ $\infty$ such that for any $0 \leq \alpha \leq 1$,

$$
C_{1} \alpha N^{2}\left(1-O\left(N^{-1}\right)\right) \leq E_{N}^{\mathcal{N}}(\alpha) \leq E_{N}^{\mathcal{D}}(\alpha) \leq C_{2} \alpha N^{2}+O(N) \text { as } N \rightarrow \infty \text {. }
$$

Moreover, in the limit $\alpha \rightarrow 0$,

$$
\liminf _{N \rightarrow \infty} \frac{E_{N}^{\mathcal{N}}(\alpha)}{N^{2}} \geq \frac{\pi}{4} \alpha\left(1-O\left(\alpha^{1 / 3}\right)\right)
$$

These results should be compared with previous results in [12] and [25], respectively. In [25] the upper bound

$$
E_{N}^{\mathcal{D}}(\alpha) / N^{2} \leq 2 \pi^{2}+O\left(N^{-1 / 2}\right) \text { independently of } \alpha
$$

was derived (the constant was not made explicit however). In [12, Theorem 1.5], lower bounds were given utilizing methods developed in [24-26] to bound the anyon interaction in terms of an effective pair interaction, which is of long range and has a coupling strength that depends on number-theoretic properties of $\alpha$. Namely, for rational $\alpha$ of the form of a reduced fraction $\alpha=\mu / v$ with $\mu, v \in \mathbb{N}, v \geq 2$ and $\mu$ odd, one defines $\alpha_{*}:=1 / \nu$, and $\alpha_{*}:=0$ otherwise. Note that $\alpha_{*}>0$ if and only if $\alpha$ is an odd-numerator rational. The result of [12, Theorem 1.5] is that

$$
\liminf _{N \rightarrow \infty} \frac{E_{N}^{\mathcal{N}}(\alpha)}{N^{2}} \geq \begin{cases}\tilde{C}_{1} \alpha_{*} & \text { for some constant } \tilde{C}_{1}>0 \\ \pi \alpha_{*}\left(1-O\left(\alpha_{*}^{1 / 3}\right)\right) & \text { as } \alpha_{*} \rightarrow 0\end{cases}
$$

While our lower bound (2.3) is weaker by a factor 4 for small $\alpha$ if $\alpha=\alpha_{*}$, it is valid for all $\alpha$, not just odd-numerator rationals.

Theorem 2.1 answers a question raised in [24,25] whether for $\alpha_{*}=0$ (and $\left.\alpha \neq 0\right)$ the energy $E_{N}^{\mathcal{N} / \mathcal{D}}(\alpha)$ could be of lower order in $N$ than the one for fermions or anyons with $\alpha_{*}>0$. It shows that the behavior of the ground-state energy is fermionic, for 
any $\alpha \neq 0$. However, it still leaves open the possibility that the exact energy in the thermodynamic limit may be smaller around even-numerator rational $\alpha$ than around $\alpha$ with relatively large $\alpha_{*}$, i.e., odd-numerator rationals with small denominator. In particular, it is not known whether it depends smoothly, or even continuously, on $\alpha$. We refer to $[1,2,20,21,25]$ for further discussion on the $\alpha$-dependence of the ground-state energy.

The improved lower bounds in Theorem 2.1 can be used to show the validity of a Lieb-Thirring inequality for anyons on the full space $\mathbb{R}^{2}$, extending the result derived in [24]. Originally, Lieb and Thirring considered fermions in the context of stability of interacting Coulomb systems [15,16] (see also [14]), and proved a uniform bound for the kinetic energy of any fermionic many-body wave function $\Psi$ in terms of an $L^{p}$-norm of its one-body density, defined as

$$
\varrho_{\Psi}(\mathbf{x}):=N \int_{\mathbb{R}^{2(N-1)}}\left|\Psi\left(\mathbf{x}, \mathbf{x}_{2}, \ldots, \mathbf{x}_{N}\right)\right|^{2} \prod_{k \geq 2} \mathrm{~d} \mathbf{x}_{k}
$$

(in fact, $p=2$ in two dimensions) thereby combining the uncertainty and Pauli exclusion principles of quantum mechanics into a single powerful bound. In [24, Theorem 1], an inequality of this type was proved to hold for anyons in case $\alpha_{*}>0$, with a quadratic dependence on $\alpha_{*}$, and was later improved in [12, Theorem 1.6] where a linear dependence in $\alpha_{*}$ was obtained. Here we extend these results to all anyons except for bosons, i.e., any $0<\alpha \leq 1$.

Theorem 2.2 (Lieb-Thirring inequality for ideal anyons) There exists a constant $C>$ 0 such that for any $0 \leq \alpha \leq 1, N \geq 1$ and $\Psi \in \mathscr{D}_{\alpha}^{N}$

$$
\sum_{j=1}^{N} \int_{\mathbb{R}^{2 N}}\left|D_{j} \Psi\right|^{2} \geq C \alpha \int_{\mathbb{R}^{2}} \varrho \Psi(\mathbf{x})^{2} \mathrm{~d} \mathbf{x}
$$

One simple consequence of Theorem 2.2 concerns the ground-state energy of $\hat{T}_{\alpha}+$ $\hat{V}$, where $\hat{V}\left(\mathbf{x}_{1}, \ldots, \mathbf{x}_{N}\right):=\sum_{j=1}^{N} V\left(\mathbf{x}_{j}\right)$ for a one-body potential $V: \mathbb{R}^{2} \rightarrow \mathbb{R}$. One gets

$$
\inf \operatorname{spec}\left(\hat{T}_{\alpha}+\hat{V}\right) \geq-\frac{1}{4 C \alpha} \int_{\mathbb{R}^{2}} V_{-}(\mathbf{x})^{2} \mathrm{~d} \mathbf{x}
$$

independently of $N$, where $V_{-}:=\max \{-V, 0\}$ denotes the negative part of $V$. Applying this, e.g., to $V(\mathbf{x})=|\mathbf{x}|^{2}-\mu$ and optimizing over $\mu>0$ gives the lower bound $\frac{4}{3} N^{3 / 2} \sqrt{C \alpha / \pi}$ on the ground-state energy of the ideal anyon gas in a harmonic oscillator potential.

The bound (2.5) may for example be applied in a physically relevant setting involving several species of charged particles subject to Coulomb interactions and confined to a very thin two-dimensional layer. Taking one of the species of particles in the layer to be anyons, as was previously considered in [26, Theorem 21], our result proves that such a system is thermodynamically stable for any type of anyon except for bosons. Our method of proof also clarifies that, at least in two dimensions, stability is a consequence solely of the local two-particle repulsive properties of any of the 
component species, in the sense that all that is required is a strictly positive energy $E_{2}^{\mathcal{N}}(\alpha)$, generalizing the Pauli exclusion principle.

\section{Upper bounds}

A key tool for obtaining upper bounds is to use the fact that interactions between particles with wave functions supported on disjoint sets can be gauged away, as described in [25]. In fact, we have the following subadditivity property for the Dirichlet energy $E_{N}^{\mathcal{D}}(\alpha ; \Omega)$ on a general domain $\Omega \subset \mathbb{R}^{2}$.

Lemma 3.1 (Subadditivity) If $\Omega_{1}$ and $\Omega_{2}$ are disjoint and simply connected subsets of $\mathbb{R}^{2}$, then

$$
E_{N_{1}+N_{2}}^{\mathcal{D}}\left(\alpha ; \Omega_{1} \cup \Omega_{2}\right) \leq E_{N_{1}}^{\mathcal{D}}\left(\alpha ; \Omega_{1}\right)+E_{N_{2}}^{\mathcal{D}}\left(\alpha ; \Omega_{2}\right)
$$

for any $0 \leq \alpha \leq 1$ and $N_{1}, N_{2} \geq 1$.

Proof Let $\Phi_{1}\left(\mathbf{x}_{1}, \ldots, \mathbf{x}_{N_{1}}\right)$ be a function in $\mathscr{D}_{\alpha}^{N_{1}}$ supported on $\Omega_{1}^{N_{1}}$, and similarly for $\Phi_{2}$ supported on $\Omega_{2}^{N_{2}}$. As a trial state for the $N_{1}+N_{2}$ particle problem, we can take

$$
\Psi(\mathrm{x})=\mathcal{S}\left[\Phi_{1}\left(\mathbf{x}_{1}, \ldots, \mathbf{x}_{N_{1}}\right) \Phi_{2}\left(\mathbf{x}_{N_{1}+1}, \ldots, \mathbf{x}_{N_{1}+N_{2}}\right) \prod_{1 \leq j \leq N_{1}<k \leq N_{1}+N_{2}} e^{-i \alpha \phi_{j k}}\right]
$$

where

$$
\phi_{j k}=\arg \frac{z_{j}-z_{k}}{\left|z_{j}-z_{k}\right|}, \quad z_{j}:=x_{j}+i y_{j},
$$

and $\mathcal{S}$ denotes symmetrization. The phase factor $\phi_{j k}$ is a priori only defined modulo $2 \pi$, but can be chosen in a smooth way for $z_{j} \in \Omega_{1}, z_{k} \in \Omega_{2}$ due to our assumptions on these domains. A simple calculation shows that

$$
\sum_{j=1}^{N_{1}+N_{2}} \int_{\left(\Omega_{1} \cup \Omega_{2}\right)^{N_{1}+N_{2}}}\left|D_{j} \Psi\right|^{2}=\sum_{j=1}^{N_{1}} \int_{\Omega_{1}^{N_{1}}}\left|D_{j}^{\prime} \Phi_{1}\right|^{2}+\sum_{j=1}^{N_{2}} \int_{\Omega_{2}^{N_{2}}}\left|D_{j}^{\prime \prime} \Phi_{2}\right|^{2}
$$

where $D_{j}^{\prime}=-i \nabla_{j}+\alpha \sum_{1 \leq k \leq N_{1}, k \neq j}\left(\mathbf{x}_{j}-\mathbf{x}_{k}\right)^{-\perp}$ and likewise for $D_{j}^{\prime \prime}$ (involving only the particles in $\Omega_{2}$ ). The claimed bound readily follows.

The following lemma gives an upper bound on $E_{N}^{\mathcal{D}}(\alpha)$ that is linear in $\alpha$ for small $\alpha$. It is restricted to small particle number, however. The bound follows from a calculation using a trial state similar to the one introduced by Dyson in [5] to obtain an upper bound on the ground-state energy of the hard-sphere Bose gas.

Lemma 3.2 (Upper bound à la Dyson) If $8 \pi \alpha N<1$, then

$$
E_{N}^{\mathcal{D}}(\alpha) \leq 2 \pi^{2} N+\frac{9 \pi}{2} N(N-1) \alpha \frac{1+\left(\frac{4}{3}\right)^{3} 20 \pi(N-2) \alpha}{(1-8 \pi \alpha N)^{2}} .
$$


Furthermore, if $2 \pi \alpha N<1$ then

$$
E_{N}^{\mathcal{N}}(\alpha) \leq 2 \pi N(N-1) \alpha \frac{1+\frac{20}{3} \pi(N-2) \alpha}{(1-2 \pi \alpha N)^{2}}
$$

Proof We choose as a trial state a real-valued function $\Phi$, in which case

$$
\sum_{j=1}^{N} \int_{Q_{0}^{N}}\left|D_{j} \Phi\right|^{2}=\sum_{j=1}^{N} \int_{Q_{0}^{N}}\left(\left|\nabla_{j} \Phi\right|^{2}+\alpha^{2}\left|\mathbf{A}_{j}\right|^{2} \Phi^{2}\right),
$$

which is the energy of an $N$-body Bose gas with two- and three-body interactions of the form

$$
\begin{aligned}
\sum_{j=1}^{N}\left|\mathbf{A}_{j}\right|^{2} & =\sum_{j=1}^{N} \sum_{k \neq j} \sum_{l \neq j}\left(\mathbf{x}_{j}-\mathbf{x}_{k}\right)^{-\perp} \cdot\left(\mathbf{x}_{j}-\mathbf{x}_{l}\right)^{-\perp} \\
& =\sum_{j \neq k}\left|\mathbf{x}_{j}-\mathbf{x}_{k}\right|^{-2}+\sum_{j \neq k \neq l \neq j}\left(\mathbf{x}_{j}-\mathbf{x}_{k}\right)^{-\perp} \cdot\left(\mathbf{x}_{j}-\mathbf{x}_{l}\right)^{-\perp} .
\end{aligned}
$$

It is well known that the minimum of the right side of (3.4) over all functions $\Phi$ is the same as the one over only bosonic $\Phi$ (see, e.g., [14, Corollary 3.1]), hence we may choose a $\Phi$ that is not permutation-symmetric. In particular, we can use a Dyson ansatz $[5,17,18]$ of the form

$$
\Phi\left(\mathbf{x}_{1}, \ldots, \mathbf{x}_{N}\right)=\prod_{j=1}^{N} \varphi\left(\mathbf{x}_{j}\right) f\left(\mathbf{x}_{j}-\mathbf{y}_{j}\left(\mathbf{x}_{j} ; \mathbf{x}_{1}, \ldots, \mathbf{x}_{j-1}\right)\right)
$$

where we take $\varphi(\mathbf{x})=2 \sin (\pi x) \sin (\pi y)$ to be the $L^{2}$-normalized ground state of the Dirichlet Laplacian on $Q_{0}, f$ is a nonnegative radial function bounded by 1 , and $\mathbf{y}_{j}\left(\mathbf{x}_{j} ; \mathbf{x}_{1}, \ldots, \mathbf{x}_{j-1}\right)$ denotes the nearest neighbor of $\mathbf{x}_{j}$ among the points $\left\{\mathbf{x}_{1}, \ldots, \mathbf{x}_{j-1}\right\}$. A straightforward generalization of the calculation in $[5,17,18]$ leads to the upper bound

$$
\begin{aligned}
E_{N}^{\mathcal{D}}(\alpha) \leq & \|\Phi\|^{-2} \sum_{j=1}^{N} \int_{Q_{0}^{N}}\left(\left|\nabla_{j} \Phi\right|^{2}+\alpha^{2}\left|\mathbf{A}_{j}\right|^{2} \Phi^{2}\right) \\
\leq & 2 \pi^{2} N+N(N-1)\|\varphi\|_{4}^{4} \frac{\int_{\mathbb{R}^{2}}|\nabla f|^{2}+\alpha^{2} \int_{B}|f(\mathbf{x})|^{2}|\mathbf{x}|^{-2}}{\left(1-N\|\varphi\|_{\infty}^{2} \int_{\mathbb{R}^{2}}\left(1-f^{2}\right)\right)^{2}} \\
& +N(N-1)(N-2)\|\varphi\|_{\infty}^{4} \frac{\frac{2}{3}\left(\int_{\mathbb{R}^{2}} f|\nabla f|\right)^{2}+\alpha^{2}\left(\int_{B}|f(\mathbf{x})|^{2}|\mathbf{x}|^{-1}\right)^{2}}{\left(1-N\|\varphi\|_{\infty}^{2} \int_{\mathbb{R}^{2}}\left(1-f^{2}\right)\right)^{2}}
\end{aligned}
$$

assuming that the term in parentheses in the denominators is strictly positive. Here $B$ denotes the ball of radius $\sqrt{2}$ centered at the origin. Note that $\|\varphi\|_{4}^{4}=9 / 4$ and $\|\varphi\|_{\infty}^{2}=4$. We shall choose 


$$
f(\mathbf{x})=\min \left\{(|\mathbf{x}| / \sqrt{2})^{\alpha}, 1\right\}
$$

in which case

$$
\int_{\mathbb{R}^{2}}|\nabla f|^{2}=\alpha^{2} \int_{B} f(\mathbf{x})^{2}|\mathbf{x}|^{-2}=\pi \alpha
$$

as well as

$$
\int_{\mathbb{R}^{2}} f|\nabla f|=\alpha \int_{B} f(\mathbf{x})^{2}|\mathbf{x}|^{-1}=\frac{\sqrt{8} \pi \alpha}{1+2 \alpha} \text { and } \int_{\mathbb{R}^{2}}\left(1-f^{2}\right)=\frac{2 \pi \alpha}{1+\alpha} .
$$

This leads to the claimed upper bound (3.2).

The same strategy can be used to obtain the upper bound (3.3) on the Neumann energy $E_{N}^{\mathcal{N}}(\alpha)$. In this case, one simply chooses $\varphi=1$ in (3.5).

A combination of Lemmas 3.1 and 3.2 leads to the following result, which immediately implies the upper bound claimed in (2.2) in Theorem 2.1.

Proposition 3.3 (Global upper bound) There exists a constant $C>0$ such that for any $0 \leq \alpha \leq 1$ and any $N \geq 1$ we have

$$
E_{N}^{\mathcal{D}}(\alpha) \leq C\left(N+\alpha N^{2}\right)
$$

Proof We shall divide the unit square $Q_{0}$ into disjoint smaller boxes and place a fixed number $n \geq 1$ particles in each box. More precisely, we divide $Q_{0}$ into $M^{2}$ smaller boxes (squares) $\left\{Q_{q}\right\}_{q=1}^{M^{2}}$ of side length $M^{-1}$, with $M=\left\lceil(N / n)^{1 / 2}\right\rceil$. We place $n$ particles into as many boxes as possible, and fewer than $n$ in the remaining ones, if necessary. Denoting the number of particles in $Q_{q}$ by $n_{q}$, and using the subadditivity in Lemma 3.1 as well as the scaling property (2.1), we obtain

$$
E_{N}^{\mathcal{D}}(\alpha) \leq M^{2} \sum_{q=1}^{M^{2}} E_{n_{q}}^{\mathcal{D}}(\alpha)
$$

We shall distinguish three cases. First, if $16 \pi \alpha \geq 1$, we shall use (3.6) for $n=1$. Since $E_{1}^{\mathcal{D}}(\alpha)=2 \pi^{2}$, we obtain

$$
E_{N}^{\mathcal{D}}(\alpha) \leq 2 \pi^{2} M^{2} N \leq 2 \pi^{2} N\left(N^{1 / 2}+1\right)^{2}
$$

In the opposite case $16 \pi \alpha<1$, we shall choose $n$ such that $8 \pi \alpha n<1$, in which case we can apply the bound of Lemma 3.2 to $E_{n_{q}}^{\mathcal{D}}(\alpha)$, and obtain

$$
E_{N}^{\mathcal{D}}(\alpha) \leq M^{2}\left(2 \pi^{2} N+\frac{9 \pi}{2} N n \alpha \frac{1+\left(\frac{4}{3}\right)^{3} 20 \pi n \alpha}{(1-8 \pi \alpha n)^{2}}\right)
$$


using $n_{q} \leq n$ on each box. Now if also $16 \pi \alpha N<1$, we take $n=N$, i.e., $M=1$, and obtain

$$
E_{N}^{\mathcal{D}}(\alpha) \leq 2 \pi^{2} N+2 \pi N^{2} \alpha\left(9+\frac{80}{3}\right)
$$

Finally, if $16 \pi \alpha<1$ and $16 \pi \alpha N \geq 1$, we take $n=\left\lfloor\frac{1}{16 \pi \alpha}\right\rfloor$ so that $16 \pi \alpha n \leq 1$. Then $M \leq\left\lceil(32 \pi \alpha N)^{1 / 2}\right\rceil \leq \frac{3}{2}(32 \pi \alpha N)^{1 / 2}$, hence

$$
E_{N}^{\mathcal{D}}(\alpha) \leq 72 \pi \alpha N^{2}\left(2 \pi^{2}+\frac{9}{8}+\frac{10}{3}\right)
$$

This completes the proof of the proposition.

\section{Lower bounds}

As in [12,24-26], the key ingredient in the strategy to obtain lower bounds is to first prove a lower bound for the local Neumann energy that is linear in the particle number $N$. By splitting the original domain suitably, one may then lift such a bound to one that is quadratic in $N$. This method and local bound, referred to as a "local exclusion principle", goes back to the way Dyson and Lenard incorporated the Pauli exclusion principle for fermions in their original proof of stability of matter [6], and was further developed in [24,26-28] for interacting bosonic gases and in [8] for a model of fermions with point interactions.

\subsection{Preliminaries}

We start by recalling some of the previously obtained lower bounds which shall also turn out to be useful in deriving the new bounds. The simplest one is the usual diamagnetic inequality which is also valid for anyons [26, Lemma 4] and tells us that their kinetic energy is always at least as big as the one of bosons:

Lemma 4.1 (Diamagnetic inequality) For any $0 \leq \alpha \leq 1, N \geq 1, \Omega \subset \mathbb{R}^{2}$ and $\Psi \in \mathscr{D}_{\alpha}^{N}$ one has the inequality

$$
\sum_{j=1}^{N} \int_{\Omega^{N}}\left|D_{j} \Psi\right|^{2} \geq \sum_{j=1}^{N} \int_{\Omega^{N}}\left|\nabla_{j}\right| \Psi||^{2}
$$

Next we consider a certain analog of Lemma 3.1 for the Neumann energy, where subadditivity becomes superadditivity.

Lemma 4.2 (Superadditivity) For $K \geq 2$, let $\left\{\Omega_{q}\right\}_{q=1}^{K}$ be a collection of disjoint, simply connected subsets of $\mathbb{R}^{2}$. For $\vec{n} \in \mathbb{N}_{0}^{K}$ with $\sum_{q} n_{q}=N$, let $\mathbb{1}_{\vec{n}}$ denote the 
characteristic function of the subset of $\mathbb{R}^{2 N}$ where exactly $n_{q}$ of the points $\left\{\mathbf{x}_{1}, \ldots, \mathbf{x}_{N}\right\}$ are in $\Omega_{q}$, for all $1 \leq q \leq K$. Let

$$
W\left(\mathbf{x}_{1}, \ldots, \mathbf{x}_{N}\right):=\sum_{\vec{n}} \sum_{q=1}^{K} E_{n_{q}}^{\mathcal{N}}\left(\alpha ; \Omega_{q}\right) \mathbb{1}_{\vec{n}}\left(\mathbf{x}_{1}, \ldots, \mathbf{x}_{N}\right)
$$

With $\Omega:=\cup_{q} \Omega_{q}$, we have

$$
\sum_{j=1}^{N} \int_{\Omega^{N}}\left|D_{j} \Psi\right|^{2} \geq \int_{\Omega^{N}} W|\Psi|^{2}
$$

for any $\Psi \in \mathscr{D}_{\alpha}^{N}$. In particular,

$$
E_{N}^{\mathcal{N}}(\alpha ; \Omega) \geq \min _{\vec{n}} \sum_{q=1}^{K} E_{n_{q}}^{\mathcal{N}}\left(\alpha ; \Omega_{q}\right)
$$

Proof We start by noting that if $\mathbf{x}_{j} \in \Omega$ for all $1 \leq j \leq N$, then $1=$ $\sum_{\vec{n}} \mathbb{1}_{\vec{n}}\left(\mathbf{x}_{1}, \ldots, \mathbf{x}_{N}\right)$. Moreover, for any given $\vec{n}$, we can further divide the support of $\mathbb{1}_{\vec{n}}$ into sets corresponding to a labeling of what particles are in what subset. Specifically, for any $\Psi \in \mathscr{D}_{\alpha}^{N}$

$$
\sum_{j=1}^{N} \int_{\Omega^{N}}\left|D_{j} \Psi\right|^{2} \mathrm{dx}=\sum_{\left\{A_{k}\right\}} \sum_{q=1}^{K} \int_{\left(\Omega \backslash \Omega_{q}\right)^{N-\left|A_{q}\right|}} \sum_{j \in A_{q}} \int_{\Omega_{q}^{\left|A_{q}\right|}}\left|D_{j} \Psi\right|^{2} \mathrm{dx}_{A_{q}} \mathrm{dx}_{A_{q}^{c}},
$$

where the sum runs over all partitions of the particles into the sets $\Omega_{q}$, i.e., over collections of disjoint subsets $A_{k} \subseteq\{1,2, \ldots, N\}$ such that $\left|A_{1}\right|+\cdots+\left|A_{K}\right|=N$. We have introduced the notation $\mathrm{dx}_{A}=\prod_{j \in A} \mathrm{~d} \mathbf{x}_{j}$. Note that, for given $q$, all the particles with labels in $A_{q}$ are located in $\Omega_{q}$, while the others are located in $\Omega \backslash \Omega_{q}$. The interaction of particles inside and outside $\Omega_{q}$ can then be gauged away, as in the proof of Lemma 3.1, explicitly by writing $\tilde{\Psi}=\prod_{j \in A_{q}, k \in A_{q}^{c}} e^{i \alpha \phi_{j k}} \Psi$, with $\phi_{j k}$ defined in $(3.1)$ :

$$
\begin{aligned}
& \sum_{j \in A_{q}} \int_{\Omega_{q}^{\left|A_{q}\right|}}\left|D_{j} \Psi\right|^{2} \mathrm{dx}_{A_{q}} \\
& =\sum_{j \in A_{q}} \int_{\Omega_{q}^{\left|A_{q}\right|}}\left|D_{j}^{\prime} \tilde{\Psi}\right|^{2} \mathrm{dx}_{A_{q}} \geq E_{\left|A_{q}\right|}^{\mathcal{N}}\left(\alpha ; \Omega_{q}\right) \int_{\Omega_{q}^{\left|A_{q}\right|}}|\tilde{\Psi}|^{2} \mathrm{dx}_{A_{q}},
\end{aligned}
$$

where $D_{j}^{\prime}=-i \nabla_{j}+\alpha \sum_{k \in A_{q}, k \neq j}\left(\mathbf{x}_{j}-\mathbf{x}_{k}\right)^{-\perp}$. Since $|\tilde{\Psi}|=|\Psi|$ we thus arrive at the desired lower bound (4.2).

With the aid of the previous two lemmas, we can obtain the following bound, which is an adaptation of [19, Proposition 2]. 
Lemma 4.3 (A priori bounds in terms of $E_{2}^{\mathcal{N}}(\alpha)$ ) For any $0 \leq \alpha \leq 1$ and $N \geq 3$ we have

$$
E_{N}^{\mathcal{N}}(\alpha) \geq \frac{\pi^{2}\left(\begin{array}{c}
N \\
2
\end{array}\right)\left(\frac{3}{4}\right)^{N-2} E_{2}^{\mathcal{N}}(\alpha)}{\left(\pi+4 \sqrt{E_{2}^{\mathcal{N}}(\alpha)}\right)^{2}+E_{2}^{\mathcal{N}}(\alpha)\left(\begin{array}{c}
N \\
2
\end{array}\right)\left(\frac{3}{4}\right)^{N-2}}
$$

Proof Let us split $Q_{0}$ into four equally large squares, $Q_{0}=Q_{1} \sqcup Q_{2} \sqcup Q_{3} \sqcup Q_{4}$. Lemma 4.2 implies that

$$
\sum_{j=1}^{N} \int_{Q_{0}^{N}}\left|D_{j} \Psi\right|^{2} \geq \int_{Q_{0}^{N}} W|\Psi|^{2}
$$

with $W$ defined in (4.1) (with $K=4$ and $\Omega_{q}=Q_{q}$ for $1 \leq q \leq 4$ ). If we keep only the terms in (4.1) where $n_{q}=2$, we obtain the lower bound

$$
W\left(\mathbf{x}_{1}, \ldots, \mathbf{x}_{N}\right) \geq W_{2}\left(\mathbf{x}_{1}, \ldots, \mathbf{x}_{N}\right):=4 E_{2}^{\mathcal{N}}(\alpha) \sum_{\vec{n}} \sum_{q=1}^{4}\left(n_{q}=2\right) \mathbb{1}_{\vec{n}}\left(\mathbf{x}_{1}, \ldots, \mathbf{x}_{N}\right)
$$

where we have introduced the convenient notation $(P)=1$ if the statement $P$ is true and $(P)=0$ otherwise, and used the scaling property $E_{2}^{\mathcal{N}}\left(\alpha ; Q_{q}\right)=4 E_{2}^{\mathcal{N}}(\alpha)$ for $1 \leq q \leq 4$. The average value of $W_{2}$ can be computed to be

$$
\int_{Q_{0}^{N}} W_{2}=E_{2}^{\mathcal{N}}(\alpha)\left(\begin{array}{l}
N \\
2
\end{array}\right)\left(\frac{3}{4}\right)^{N-2}
$$

by counting the probability that exactly two particles are in a given square.

In order to estimate the expectation value of the potential $W_{2}$ in a ground state $\Psi$, we borrow a bit of kinetic energy and use the diamagnetic inequality of Lemma 4.1. That is, for arbitrary $\kappa \in[0,1]$ we write

$$
\hat{T}_{\alpha}^{Q_{0}, \mathcal{N}}=\kappa \hat{T}_{\alpha}^{Q_{0}, \mathcal{N}}+(1-\kappa) \hat{T}_{\alpha}^{Q_{0}, \mathcal{N}} \geq \kappa \hat{T}_{\alpha}^{Q_{0}, \mathcal{N}}+(1-\kappa) W_{2}
$$

The diamagnetic inequality then implies that

$$
E_{N}^{\mathcal{N}}(\alpha)=\inf \operatorname{spec} \hat{T}_{\alpha}^{Q_{0}, \mathcal{N}} \geq \inf \operatorname{spec}\left[-\kappa \Delta_{Q_{0}^{N}}^{\mathcal{N}}+(1-\kappa) W_{2}\right]
$$

with $\Delta_{Q_{0}^{N}}^{\mathcal{N}}$ denoting the Neumann Laplacian on $Q_{0}^{N}$. Consider the projection $P_{0}:=$ $u_{0}\left\langle u_{0}, \cdot\right\rangle$ onto its normalized ground state $u_{0} \equiv 1$, and the orthogonal complement $P_{0}^{\perp}=\mathbb{1}-P_{0}$, for which we have

$$
-\Delta_{Q_{0}^{N}}^{\mathcal{N}} \geq \pi^{2} P_{0}^{\perp}
$$


Since $W_{2} \geq 0$, the Cauchy-Schwarz inequality implies that

$$
W_{2}=\left(P_{0}+P_{0}^{\perp}\right) W_{2}\left(P_{0}+P_{0}^{\perp}\right) \geq(1-\varepsilon) P_{0} W_{2} P_{0}+\left(1-\varepsilon^{-1}\right) P_{0}^{\perp} W_{2} P_{0}^{\perp},
$$

for arbitrary $\varepsilon \in(0,1)$. We have

$$
P_{0} W_{2} P_{0}=P_{0} \int_{Q_{0}^{N}} W_{2}
$$

By using also the simple bound

$$
P_{0}^{\perp} W_{2} P_{0}^{\perp} \leq P_{0}^{\perp}\left\|W_{2}\right\|_{\infty} \leq P_{0}^{\perp} 2^{4} E_{2}^{\mathcal{N}}(\alpha),
$$

we obtain

$$
\begin{aligned}
-\kappa \Delta_{Q_{0}^{N}}^{\mathcal{N}}+(1-\kappa) W_{2} \geq & \left(\kappa \pi^{2}-(1-\kappa)\left(\varepsilon^{-1}-1\right) 2^{4} E_{2}^{\mathcal{N}}(\alpha)\right) P_{0}^{\perp} \\
& +(1-\kappa)(1-\varepsilon)\left(\begin{array}{c}
N \\
2
\end{array}\right)\left(\frac{3}{4}\right)^{N-2} E_{2}^{\mathcal{N}}(\alpha) P_{0}
\end{aligned}
$$

The optimal choice of $\kappa$ is to make the prefactors in front of the two projections on the right side equal, i.e.,

$$
\kappa=\frac{2^{4}\left(\varepsilon^{-1}-1\right) E_{2}^{\mathcal{N}}(\alpha)\left[1+\varepsilon\left(\begin{array}{c}
N \\
2
\end{array}\right) \frac{3^{N-2}}{4^{N}}\right]}{\pi^{2}+2^{4}\left(\varepsilon^{-1}-1\right) E_{2}^{\mathcal{N}}(\alpha)\left[1+\varepsilon\left(\begin{array}{c}
N \\
2
\end{array}\right) \frac{3^{N-2}}{4^{N}}\right]} .
$$

This choice leads to the bound

$$
E_{N}^{\mathcal{N}}(\alpha) \geq \frac{\pi^{2}(1-\varepsilon)\left(\begin{array}{c}
N \\
2
\end{array}\right)\left(\frac{3}{4}\right)^{N-2} E_{2}^{\mathcal{N}}(\alpha)}{\pi^{2}+2^{4}\left(\varepsilon^{-1}-1\right) E_{2}^{\mathcal{N}}(\alpha)\left[1+\varepsilon\left(\begin{array}{c}
N \\
2
\end{array}\right) \frac{3^{N-2}}{4^{N}}\right]}
$$

Optimizing over $0<\varepsilon<1$ then yields the claimed bound.

Remark 4.4 Lemma 4.3 implies, in particular, that $E_{N}^{\mathcal{N}}(\alpha)$ is bounded below by a strictly positive, $N$-dependent constant times $E_{2}^{\mathcal{N}}(\alpha)$. In fact, by localizing the two particles in different halves of the unit square $Q_{0}$ (following the proof of Lemma 3.1), one readily checks that $E_{2}^{\mathcal{N}}(\alpha) \leq 2 \pi^{2}$ independently of $\alpha$. Using this in the denominator in (4.4) leads to the simpler (but worse) bound

$$
E_{N}^{\mathcal{N}}(\alpha) \geq \frac{\left(\begin{array}{c}
N \\
2
\end{array}\right)\left(\frac{3}{4}\right)^{N-2}}{(1+4 \sqrt{2})^{2}+2\left(\begin{array}{c}
N \\
2
\end{array}\right)\left(\frac{3}{4}\right)^{N-2}} E_{2}^{\mathcal{N}}(\alpha)
$$


Note that while this gives a nonzero bound for all $N \geq 2$, the constant appearing on the right side is exponentially small as $N \rightarrow \infty$. Moreover, from (3.3) we deduce that $E_{2}^{\mathcal{N}}(\alpha) \leq 4 \pi \alpha(1+O(\alpha))$ for small $\alpha$, hence (4.4) implies that

$$
E_{N}^{\mathcal{N}}(\alpha) \geq\left(\begin{array}{l}
N \\
2
\end{array}\right)\left(\frac{3}{4}\right)^{N-2} E_{2}^{\mathcal{N}}(\alpha)(1-O(\sqrt{\alpha}))
$$

for small $\alpha$.

As a final step in this subsection, we shall give a lower bound on $E_{2}^{\mathcal{N}}(\alpha)$. The following bound is actually contained in [12, Lemma 5.3].

Lemma 4.5 (Lower bound on $E_{2}^{\mathcal{N}}(\alpha)$ ) For $v>0$ let $j_{v}^{\prime}$ denote the first positive zero of the derivative of the Bessel function $J_{v}$, satisfying

$$
\sqrt{2 v} \leq j_{v}^{\prime} \leq \sqrt{2 v(1+v)}
$$

and $j_{0}^{\prime}:=0$ for continuity. There exists a function $f:\left[0,\left(j_{1}^{\prime}\right)^{2}\right] \rightarrow \mathbb{R}_{+}$satisfying

$$
t / 6 \leq f(t) \leq 2 \pi t \quad \text { and } \quad f(t)=2 \pi t\left(1-O\left(t^{1 / 3}\right)\right) \text { as } t \rightarrow 0,
$$

such that

$$
E_{2}^{\mathcal{N}}(\alpha) \geq f\left(\left(j_{\alpha}^{\prime}\right)^{2}\right)
$$

holds for any $0 \leq \alpha \leq 1$.

In fact, the function $f$ in Lemma 4.5 is defined as

$$
\begin{aligned}
& f(t):=\frac{1}{2} \sup _{\kappa \in(0,1)} \inf _{Q_{0}^{2}|\psi|^{2}=1} \int_{Q_{0}^{2}}\left(\kappa\left(\left|\nabla_{1}\right| \psi||^{2}+\left|\nabla_{2}\right| \psi||^{2}\right)\right. \\
& \left.+(1-\kappa) t \frac{\mathbb{1}_{B_{\delta(\mathbf{X})}}(\mathbf{r})}{\delta(\mathbf{X})^{2}}|\psi|^{2}\right) \mathrm{d} \mathbf{x}_{1} \mathrm{~d} \mathbf{x}_{2}
\end{aligned}
$$

where $B_{r}$ denotes the ball of radius $r$ centered at the origin, and

$$
\mathbf{r}=\left(\mathbf{x}_{1}-\mathbf{x}_{2}\right) / 2, \quad \mathbf{X}=\left(\mathbf{x}_{1}+\mathbf{x}_{2}\right) / 2, \quad \delta(\mathbf{x}):=\operatorname{dist}\left(\mathbf{x}, \partial Q_{0}\right)
$$

Note that in combination with the upper bound (3.3) of Lemma 3.2, Lemma 4.5 determines the two-particle energy for small $\alpha$ :

Proposition 4.6 For the 2-anyon Neumann energy

$$
E_{2}^{\mathcal{N}}(\alpha)=4 \pi \alpha\left(1+O\left(\alpha^{1 / 3}\right)\right) \quad \text { as } \alpha \rightarrow 0 .
$$


Remark 4.7 The bound in [12, Lemma 5.3] is actually more general than what is stated here. It gives a lower bound, for any $N \geq 2$, in terms of the 'fractionality' of $\alpha$ [24, Proposition 5] defined as

$$
\alpha_{N}:=\min _{p \in\{0,1, \ldots, N-2\}} \min _{q \in \mathbb{Z}}|(2 p+1) \alpha-2 q|, \quad \alpha_{*}=\inf _{N \geq 2} \alpha_{N}=\lim _{N \rightarrow \infty} \alpha_{N} .
$$

Note that $\alpha_{2}=\alpha$ for $0 \leq \alpha \leq 1$. One has, in fact, for any $\alpha \in \mathbb{R}$ and $N \geq 1$ the bound

$$
E_{N}^{\mathcal{N}}(\alpha) \geq f\left(\left(j_{\alpha_{N}}^{\prime}\right)^{2}\right)(N-1)_{+}
$$

For $\alpha_{*}>0$, the right side grows linearly in $N$.

\subsection{New bounds}

Our improved lower bounds are due to the following lemma, which utilizes the scale invariance of the problem:

Lemma 4.8 ( $N$-linear bound in terms of few-particle energies) For any $0 \leq \alpha \leq 1$ and $N \geq 2$ we have

$$
E_{N}^{\mathcal{N}}(\alpha) \geq \frac{N}{4} \min \left\{E_{2}^{\mathcal{N}}(\alpha), E_{3}^{\mathcal{N}}(\alpha), E_{4}^{\mathcal{N}}(\alpha)\right\}
$$

Proof Without loss of generality we can assume $N \geq 5$, since for $N \in\{2,3,4\}$ the bound (4.6) trivially holds. We may also assume $\alpha>0$, so that $E_{N}^{\mathcal{N}}(\alpha)>0$ for all $N \geq 2$ by Lemmas 4.3 and 4.5. Let us proceed similarly as in the proof of Lemma 4.3 and split $Q_{0}=Q_{1} \sqcup Q_{2} \sqcup Q_{3} \sqcup Q_{4}$ into four equally large squares. The bound (4.3) together with the scaling property (2.1) implies

$$
E_{N}^{\mathcal{N}}(\alpha) \geq 4 \min _{\vec{n}} \sum_{q=1}^{4} E_{n_{j}}^{\mathcal{N}}(\alpha) .
$$

For any partition $\vec{n}$ of the $N$ particles into the four squares there must be at least one square with at least $N / 4$ particles. Dropping the other terms, we thus obtain the recursive bound

$$
E_{N}^{\mathcal{N}}(\alpha) \geq 4 \min _{k=\lceil N / 4\rceil, \ldots, N} E_{k}^{\mathcal{N}}(\alpha)
$$

Let us define, for $k \geq 0$,

$$
e_{k}:=\min _{n=4^{k}+1,4^{k}+2, \ldots, 4^{k+1}} E_{n}^{\mathcal{N}}(\alpha)
$$

and observe that by (4.7)

$e_{k} \geq 4 \min _{n=4^{k}+1, \ldots, 4^{k+1}} \min _{p=\lceil n / 4\rceil, \ldots, n} E_{p}^{\mathcal{N}}(\alpha) \geq 4 \min _{n=4^{k-1}+1, \ldots, 4^{k+1}} E_{n}^{\mathcal{N}}(\alpha)=4 \min \left\{e_{k-1}, e_{k}\right\}$ 
for any $k \geq 1$. Then, since $e_{k}>0$ for all $k$, we have

$$
e_{k} \geq 4 e_{k-1} \geq \cdots \geq 4^{k} e_{0}
$$

Finally, writing any $N \geq 5$ uniquely as $N=4^{k}+l$ with $1 \leq l \leq 3 \cdot 4^{k}$, we have $k \geq 1, N \leq 4^{k+1}$, and

$$
E_{N}^{\mathcal{N}}(\alpha) \geq e_{k} \geq 4^{k} e_{0} \geq \frac{N}{4} e_{0}
$$

with $e_{0}=\min \left\{E_{2}^{\mathcal{N}}(\alpha), E_{3}^{\mathcal{N}}(\alpha), E_{4}^{\mathcal{N}}(\alpha)\right\}$. This proves the statement of the lemma.

The previous lemma gives a lower bound on $E_{N}^{\mathcal{N}}(\alpha)$ that is linear in $N$, at least for $N \geq 2$, for all $\alpha>0$. The following bound (which also appeared in slightly different formulations in the earlier works; see $[8,12,19])$ lifts any linear growth in the particle number $N$ to a quadratic one.

Lemma 4.9 (Quadratic bounds) If there exists an integer $k \geq 1$ and a function $c(\alpha) \geq$ 0 such that $E_{N}^{\mathcal{N}}(\alpha) \geq c(\alpha)(N-k)_{+}$for all $N \geq 1$, then in fact

$$
E_{N}^{\mathcal{N}}(\alpha) \geq c(\alpha) \frac{N^{2}}{4 k}(1-O(k / N)) \text { as } N \rightarrow \infty
$$

Proof Given an integer $K \geq 1$, we split $Q_{0}$ into $K^{2}$ disjoint and equally large squares $\left\{Q_{q}\right\}_{q=1}^{K^{2}}$, and associate with any $L^{2}$-normalized symmetric wave function $\Psi$ the probabilities

$$
p_{n}(q):=\left(\begin{array}{l}
N \\
n
\end{array}\right) \int_{\left(Q_{q}^{c}\right)^{N-n} \times Q_{q}^{n}}|\Psi|^{2}
$$

of finding exactly $n$ particles on a square $Q_{q}$. Lemma 4.2 implies that

$$
\begin{aligned}
\sum_{j=1}^{N} \int_{Q_{0}^{N}}\left|D_{j} \Psi\right|^{2} & \geq \sum_{q=1}^{K^{2}} \sum_{n=0}^{N} E_{n}^{\mathcal{N}}\left(\alpha ; Q_{q}\right) p_{n}(q)=K^{2} \sum_{q=1}^{K^{2}} \sum_{n=0}^{N} E_{n}^{\mathcal{N}}(\alpha) p_{n}(q) \\
& \geq K^{2} \sum_{q=1}^{K^{2}} \sum_{n=0}^{N} c(\alpha)(n-k)_{+} p_{n}(q)=c(\alpha) K^{4} \sum_{n=0}^{N}(n-k)_{+} \gamma_{n}
\end{aligned}
$$

where the average distribution of particle numbers $\gamma_{n}:=K^{-2} \sum_{q=1}^{K^{2}} p_{n}(q)$ satisfies

$$
\sum_{n=0}^{N} \gamma_{n}=1 \quad \text { and } \quad \sum_{n=0}^{N} n \gamma_{n}=N / K^{2}=: \rho_{Q}
$$


the expected number of particles on any square. Hence, by convexity of $x \mapsto(x-k)_{+}$,

$$
\sum_{j=1}^{N} \int_{Q_{0}^{N}}\left|D_{j} \Psi\right|^{2} \geq c(\alpha) K^{4}\left(\sum_{n=0}^{N} n \gamma_{n}-k\right)_{+}=c(\alpha) N^{2} \rho_{Q}^{-2}\left(\rho_{Q}-k\right)_{+} \cdot
$$

In order to maximize the right side, the optimal choice of $K$ would be such as to make $\rho_{Q}=2 k$, in which case the desired bound would be obtained exactly. However, we have to take into account the constraint that $\rho_{Q}=N / K^{2}$ with $K \in \mathbb{N}$. Thus, taking $K:=\lceil\sqrt{N /(2 k)}\rceil$ we obtain

$$
\frac{2 k}{(1+\sqrt{2 k / N})^{2}} \leq \rho_{Q} \leq 2 k
$$

and

$$
E_{N}^{\mathcal{N}}(\alpha) \geq c(\alpha) \frac{N^{2}}{4 k}\left(2(1+\sqrt{2 k / N})^{2}-(1+\sqrt{2 k / N})^{4}\right)_{+},
$$

which proves the lemma.

The proof of the lower bounds of Theorem 2.1 now follows in a straightforward manner. For any $\alpha \in \mathbb{R}$ and $N \geq 2$, we have by Lemma 4.8

$$
E_{N}^{\mathcal{N}}(\alpha) \geq c(\alpha) N \quad \text { with } \quad c(\alpha):=\frac{1}{4} \min \left\{E_{2}^{\mathcal{N}}(\alpha), E_{3}^{\mathcal{N}}(\alpha), E_{4}^{\mathcal{N}}(\alpha)\right\}
$$

In particular,

$$
E_{N}^{\mathcal{N}}(\alpha) \geq c(\alpha)(N-1)_{+}
$$

for all $N \geq 1$, and therefore by Lemma 4.9

$$
E_{N}^{\mathcal{N}}(\alpha) \geq \frac{c(\alpha)}{4} N^{2}\left(1-O\left(N^{-1}\right)\right)
$$

for large $N$.

From Lemma 4.3, one can deduce that

$$
c(\alpha) \geq \frac{1}{4} \min \left\{E_{2}^{\mathcal{N}}(\alpha), 0.147\right\}
$$

where the number 0.147 is really the positive root of $(\pi+4 \sqrt{x})^{2}+\frac{9}{4} x=\frac{9}{4} \pi^{2}$, i.e.,

$$
x=\pi^{2} \frac{877-96 \sqrt{69}}{5329} \approx 0.147
$$

In combination with Lemma 4.5 and (4.5), this concludes the proof. 


\subsection{Lieb-Thirring inequality}

Finally, we explain how the above bounds lead to improvements in the local exclusion principle and thus the Lieb-Thirring inequality introduced for anyons in [24]. Namely, define for any $L^{2}$-normalized $N$-anyon wave function $\Psi \in \mathscr{D}_{\alpha}^{N}$ and domain $\Omega \subseteq \mathbb{R}^{2}$ the local kinetic energy on $\Omega$

$$
T_{\alpha}^{\Omega}[\Psi]:=\sum_{j=1}^{N} \int_{\mathbb{R}^{d N}}\left|D_{j} \Psi\right|^{2} \mathbb{1}_{\Omega}\left(\mathbf{x}_{j}\right) \mathrm{d} \mathbf{x}_{1} \cdots \mathrm{d} \mathbf{x}_{N},
$$

where $\mathbb{1}_{\Omega}$ denotes the characteristic function of $\Omega$. Applying the bound (4.8)-(4.9) as in [24, Lemma 8] we then obtain the following:

Lemma 4.10 (Local exclusion principle) For any square $Q \subset \mathbb{R}^{2}$, any $N \geq 1$ and $L^{2}$-normalized $\Psi \in \mathscr{D}_{\alpha}^{N}$ with one-particle density $\rho_{\Psi}$ (defined in (2.4)), we have

$$
T_{\alpha}^{Q}[\Psi] \geq \frac{c(\alpha)}{|Q|}\left(\int_{Q} \varrho_{\Psi}(\mathbf{x}) \mathrm{d} \mathbf{x}-1\right)_{+},
$$

where $c(\alpha):=\frac{1}{4} \min \left\{E_{2}^{\mathcal{N}}(\alpha), E_{3}^{\mathcal{N}}(\alpha), E_{4}^{\mathcal{N}}(\alpha)\right\}$ satisfies $(4.10)$.

By applying the method of [24] (see also [20] for a more detailed exposition), replacing [24, Lemma 8 ] by the above bound and using (4.10) and Lemma 4.5, one directly obtains the Lieb-Thirring inequality of Theorem 2.2 for some universal constant $C>0$.

Acknowledgements D. L. would like to thank Simon Larson for discussions. Financial support from the Swedish Research Council, Grant No. 2013-4734 (D. L.), the European Research Council (ERC) under the European Union's Horizon 2020 research and innovation programme (Grant Agreement No 694227, R. S.), and by the Austrian Science Fund (FWF), project Nr. P 27533-N27 (R. S.), is gratefully acknowledged.

Open Access This article is distributed under the terms of the Creative Commons Attribution 4.0 International License (http://creativecommons.org/licenses/by/4.0/), which permits unrestricted use, distribution, and reproduction in any medium, provided you give appropriate credit to the original author(s) and the source, provide a link to the Creative Commons license, and indicate if changes were made.

\section{References}

1. Correggi, M., Lundholm, D., Rougerie, N.: Local density approximation for almost-bosonic anyons. In: Proceedings of QMath13, Atlanta, October 8-11, 2016 (2017). arXiv:1705.03203 (to appear)

2. Correggi, M., Lundholm, D., Rougerie, N.: Local density approximation for the almost-bosonic anyon gas. Anal. PDE 10, 1169-1200 (2017). https://doi.org/10.2140/apde.2017.10.1169

3. Date, G., Murthy, M.V.N., Vathsan, R.: Classical and quantum mechanics of anyons, arXiv e-prints (2003). arXiv:cond-mat/0302019

4. Dell'Antonio, G., Figari, R., Teta, A.: Statistics in space dimension two. Lett. Math. Phys. 40(3), 235-256 (1997). https://doi.org/10.1023/A:1007361832622

5. Dyson, F.J.: Ground-state energy of a hard-sphere gas. Phys. Rev. 106(1), 20-26 (1957). https://doi. org/10.1103/PhysRev.106.20

6. Dyson, F.J., Lenard, A.: Stability of matter. I. J. Math. Phys. 8(3), 423-434 (1967). https://doi.org/10. $1063 / 1.1705209$ 
7. Forte, S.: Quantum mechanics and field theory with fractional spin and statistics. Rev. Mod. Phys. 64, 193-236 (1992). https://doi.org/10.1103/RevModPhys.64.193

8. Frank, R.L., Seiringer, R.: Lieb-Thirring inequality for a model of particles with point interactions. J. Math. Phys 53(9), 095201, 11 (2012). https://doi.org/10.1063/1.3697416

9. Fröhlich, J.: Quantum statistics and locality. In: Proceedings of the Gibbs Symposium (New Haven, CT, 1989), pp. 89-142. American Mathematical Society, Providence (1990)

10. Iengo, R., Lechner, K.: Anyon quantum mechanics and Chern-Simons theory. Phys. Rep. 213, 179-269 (1992). https://doi.org/10.1016/0370-1573(92)90039-3

11. Khare, A.: Fractional Statistics and Quantum Theory, 2nd edn. World Scientific, Singapore (2005)

12. Larson, S., Lundholm, D.: Exclusion bounds for extended anyons. Arch. Ration. Mech. Anal. 227, 309-365 (2017). https://doi.org/10.1007/s00205-017-1161-9

13. Lerda, A.: Anyons. Springer, Berlin (1992)

14. Lieb, E.H., Seiringer, R.: The Stability of Matter in Quantum Mechanics. Cambridge University Press, Cambridge (2010)

15. Lieb, E.H., Thirring, W.E.: Bound for the kinetic energy of fermions which proves the stability of matter. Phys. Rev. Lett. 35, 687-689 (1975). https://doi.org/10.1103/PhysRevLett.35.687

16. Lieb, E.H., Thirring, W.E.: Inequalities for the moments of the eigenvalues of the Schrödinger hamiltonian and their relation to Sobolev inequalities. Studies in Mathematical Physics. Princeton University Press, Princeton (1976)

17. Lieb, E.H., Yngvason, J.: The ground state energy of a dilute two-dimensional Bose gas. J. Stat. Phys. 103(3-4), 509-526 (2001). https://doi.org/10.1023/A:1010337215241

18. Lieb, E.H., Seiringer, R., Yngvason, J.: Bosons in a trap: a rigorous derivation of the Gross-Pitaevskii energy functional. Phys. Rev. A 61(4), 043602 (2000). https://doi.org/10.1103/PhysRevA.61.043602

19. Lundholm, D.: Anyon wave functions and probability distributions, IHÉS preprint, IHES/P/13/25, (2013). http://preprints.ihes.fr/2013/P/P-13-25.pdf

20. Lundholm, D.: Methods of modern mathematical physics: uncertainty and exclusion principles in quantum mechanics, Lecture notes, KTH (2017). arXiv:1805.03063

21. Lundholm, D.: Many-anyon trial states. Phys. Rev. A 96, 012116 (2017). https://doi.org/10.1103/ PhysRevA.96.012116

22. Lundholm, D., Rougerie, N.: The average field approximation for almost bosonic extended anyons. J. Stat. Phys. 161(5), 1236-1267 (2015). https://doi.org/10.1007/s10955-015-1382-y

23. Lundholm, D., Rougerie, N.: Emergence of fractional statistics for tracer particles in a Laughlin liquid. Phys. Rev. Lett. 116, 170401 (2016). https://doi.org/10.1103/PhysRevLett.116.170401

24. Lundholm, D., Solovej, J.P.: Hardy and Lieb-Thirring inequalities for anyons. Commun. Math. Phys. 322, 883-908 (2013). https://doi.org/10.1007/s00220-013-1748-4

25. Lundholm, D., Solovej, J.P.: Local exclusion principle for identical particles obeying intermediate and fractional statistics. Phys. Rev. A 88, 062106 (2013). https://doi.org/10.1103/PhysRevA.88.062106

26. Lundholm, D., Solovej, J.P.: Local exclusion and Lieb-Thirring inequalities for intermediate and fractional statistics. Ann. Henri Poincaré 15, 1061-1107 (2014). https://doi.org/10.1007/s00023-0130273-5

27. Lundholm, D., Portmann, F., Solovej, J.P.: Lieb-Thirring bounds for interacting Bose gases. Commun. Math. Phys. 335(2), 1019-1056 (2015). https://doi.org/10.1007/s00220-014-2278-4

28. Lundholm, D., Nam, P.T., Portmann, F.: Fractional Hardy-Lieb-Thirring and related inequalities for interacting systems. Arch. Ration. Mech. Anal. 219(3), 1343-1382 (2016). https://doi.org/10.1007/ s00205-015-0923-5

29. Myrheim, J.: Topological aspects of low dimensional systems. In: Comtet, A., Jolicœur, T., Ouvry, S., David, F. (eds.) Les Houches_Ecole d'Ete de Physique Theorique, Anyons, vol. 69, pp. 265-413. Springer, Berlin (1999). https://doi.org/10.1007/3-540-46637-1_4

30. Nayak, C., Simon, S.H., Stern, A., Freedman, M., Sarma, S.D.: Non-abelian anyons and topological quantum computation. Rev. Mod. Phys. 80, 1083-1159 (2008). https://doi.org/10.1103/RevModPhys. 80.1083

31. Ouvry, S.: Anyons and lowest Landau level anyons. Séminaire Poincaré 11, 77-107 (2007). https:// doi.org/10.1007/978-3-7643-8799-0_3

32. Stern, A.: Anyons and the quantum Hall effect-a pedagogical review. Ann. Phys. 323(1), 204-249 (2008). https://doi.org/10.1016/j.aop.2007.10.008. (January Special Issue 2008)

33. Wilczek, F.: Fractional Statistics and Anyon Superconductivity. World Scientific, Singapore (1990) 